\title{
A Secure Base From Which To Explore Close Relationships
}

\author{
Everett Waters \\ State University of New York at Stony Brook \\ E. Mark Cummings \\ Notre Dame University
}

\begin{abstract}
The theory of attachment as a secure base relationship integrates insights about affect, cognition, and behavior in close relationships across age and culture. Its empirical successes include important discoveries about the nature of infant-caregiver and adult-adult close relationships, the importance of early experience, and about stability and change in individual differences. The task now is to preserve these insights and successes and build on them. To accomplish this, we need to continually examine the logic and coherence of attachment theory and redress errors of emphasis and errors of analysis. Views on attachment development, attachment representation, and attachment in family and cross-cultural perspective require updating in light of empirical research and advances in developmental theory, behavioral biology, and cognitive psychology. We also need to challenge the theory by formulating and testing hypotheses which, if not confirmed, would require significant changes to the theory. If we can accomplish these tasks, prospects for important developments in attachment theory and research are greater than ever, as are the prospects for integration with other disciplines.
\end{abstract}

For attachment theorists and researchers, the first year of this new century also marks the first year of attachment study without both John Bowlby and Mary Ainsworth. Of course, we are not entirely without them. The BowlbyAinsworth attachment theory is a valuable legacy, and can continue to serve as a secure base from which to explore close relationships. This reflects (1) the value of Freud's insights about the nature and importance of early rela- tionships, (2) Bowlby's wisdom in reconceptualizing the infant-mother tie as a secure base relationship, (3) the health and progress of control systems theory, evolutionary theory, and cognitive psychology, the sciences that underpin attachment theory, and (4) the lasting value of Ainsworth's initial ethological studies of infant attachment in Uganda and Baltimore. The task now is to enrich this legacy by rigorous theoretical and empirical analysis.

The authors would like to thank Judith Crowell, Melanie Elliot, Dominique Treboux, Brian Vaughn, Polly Vittorini, and Harriet Waters for valuable editorial comments. Preparation of this paper was supported in part by grants from the National Institute of Mental Health (MH 44935) to Everett Waters and Judith Crowell and from the National Institute of Child Health and Human Development (HD 36261) to Mark Cummings. Correspondence regarding this article should be addressed to Dr. Everett Waters, Department of Psychology, SUNY, Stony Brook, NY 11794-2500 or Dr. E. Mark Cummings, Department of Psychology, Notre Dame University, 215 Haggar Hall, Notre Dame, IN 46556. 


\section{The State of The Art}

Advances in attachment theory have introduced productive new ideas about early relationship experience (e.g., Bretherton \& Mulholland, 1999; Cummings \& Davies, 1996). Building on these ideas, advances in assessment (e.g.; Crowell \& Treboux, 1995; Davies \& Cummings, 1998; Waters, 1997) have led to an explosion of research on attachment beyond infancy (e.g., Greenberg, Cicchetti, \& Cummings, 1990). Infants whose attachment security was assessed in the late 1970's are becoming young adults, and thus offering first opportunities to test key hypotheses about relations between infant attachment and adult love relationships (e.g., Waters, Hamilton, \& Weinfield, 1999).

Maintaining the coherence and empirical underpinnings of attachment theory is a continuous process of updating key ideas in light of advances in theory, data, and other areas of psychology, and subjecting the theory to severe tests - test which, if not passed, would require us to reject the theory or at least make significant revisions and influence subsequent research (viz. Mayo, 1996; Meehl, 1978; Smedslund, 1994).

For attachment theory a significant challenge is to assimilate so many advances and remain coherent. The greatest risk is resting on the laurels earned by past successes - foregoing Bowlby's commitment to rigorous theoretical and empirical analysis, and settling for research that can at best confirm what we already know. Accordingly, this essay proposes (1) keeping the underlying logic of Bowlby's theory and the secure base concept at center stage in attachment theory and research, (2) updating traditional views of attachment development, function, and working models, and (3) looking to family, life span, and cross-cultural contexts to test key postulates of attachment theory.

\section{A Secure Base From Which To Explore}

Like a secure attachment, a well formed scientific theory encourages exploration, organizes experience, and helps one work effectively under uncertainty. In an era dominated by domain specific theory, the Bowlby-Ainsworth tra- dition is a rare example of a more general or "grand theory" that makes predictions about behavior and emotion across multiple domains of psychological functioning and across the life span. Accordingly, the historical origins and underlying logic of attachment theory, which are rarely considered, merit examination.

Bowlby realized sooner than many that a paradigm shift was sweeping psychoanalytic theory from the mainstream of scientific psychology. He also realized that we might, in the process, discard genuine insights infant-mother and adult-adult relationships. Thus a primary goal in developing modern attachment theory was to preserve the kernels of truth in Freud's insights about close relationships by replacing his image of a needy, dependent infant motivated by drive reduction with one of a sophisticated, competence-motivated infant using its primary caregiver as a secure base from which to explore and, when necessary, as a haven of safety and a source of comfort. ${ }^{1}$ Within this framework, the child's tie to its mother reflects the operating characteristics of an underlying control system that collates information about the infant's state, the state of the environment, and past and current access to the caregiver. Bowlby introduced the term attachment to refer specifically to this secure base formulation of infant-adult and adult-adult ties and to distinguish it from psychodynamic and learning theory perspectives (viz. Ainsworth, 1969). It is neither a generic term not an exhaustive perspective on human relationships.

Devoted to rigorous analysis, Bowlby realized that replacing Freud's drive theory with an attachment control-system would amount to little more that replacing one kind of magic with another unless he could explain the origin and nature of behavioral control-systems. For this, he turned to evolutionary theory, arguing from many examples that such control-systems reflect species specific biases in learning abilities that can be shaped by evolution. The attachment control system is constructed during development through interaction between biases in our learning abilities and experience with caregivers 
and environments. These biases in our learning abilities are part of our primate heritage and are available to every human infant. Attachment control systems are constructed through experience, not pre-wired, and are hypothesized to play a role in the organization of behavior and emotion in close relationships throughout the life span.

Keeping the Secure Base Concept at Center Stage in Attachment Theory and Research

The secure base concept is central to the logic and coherence of attachment theory and to its status as an organizational construct. For both Bowlby and Ainsworth, to be attached is to use someone preferentially as a secure base form which to explore. The term secure attachment refers both to skillful secure base use over time and contexts in naturalistic settings and to confidence in a caregiver's availability and responsiveness. Within this framework, naturalistic observations of secure base behavior are the gold standard against which attachment measures are validated and against which they are must be revalidated for use in different age groups, research populations, and cultures (Ainsworth, Blehar, Waters, \& Wall, 1978; Waters, KondoIkemura, Posada, \& Richters, (1991).

It is surprising therefore to find the secure base concept increasingly removed from center stage in current theory and research, displaced by cognitive constructs such as working models and psychometric constructs such as anxiety and avoidance. Clearly, for Bowlby the working models concept represented an insight into the mental representation of appraisal and set goal components of the secure base control system, not a new formulation to replace them. In fact, ideas about the representation of early experience play an important role in applying the secure base concept to attachment relationships after infancy. However, without the constraints imposed by the secure base control systems framework, the working models concept and constructs such as anxiety and avoidance, become too loosely defined and offer to explain too much.

It is also surprising that the secure base concept is often overlooked as a criterion against which to validate new attachment measures or traditional measures when they are to be used in new age groups, at-risk samples, or across cultures. Over time, this can erode the coherence of attachment theory and research.

Insights into the nature of attachment representations and advances in measurement are always welcome. But it is important to clearly define their links to the secure base concept. It is central to the logic of key insights about early experience and close relationships. Unless we want to discard these insights or develop alternative explanations, logic requires that we keep the secure base concept at center stage in attachment theory and research.

\section{Securing Key Concepts}

\section{Attachment Development}

A detailed developmental analysis is integral to attachment theory. Bowlby's (1969) developmental outline included four phases: undiscriminating social responsiveness (0-3 months), preferential social responsiveness (3-6 months), emergence of secure base behavior (6-24 months), and goal corrected partnership (24-30 + months). Although this outline has served well, it was not meant to be the last word. In particular, it provided much more detail about the origins and onset of attachment behavior than about its fate after infancy. In part, the data at hand provided better coverage of early infancy. In addition, Bowlby emphasized the early phases because accounting for the onset and organization of attachment behavior was critical to explaining his new theory.

Unfortunately, Bowlby's emphasis on the early phase of attachment development has been a source of misunderstandings and missed opportunities. Misunderstandings because it suggests that secure base behavior emerges rather quickly, implying to some that learning and socialization play little part in his model. Missed opportunities because it doesn't direct attention to the maintaining and shaping influence of caregiver behavior or developmental changes in secure base use beyond infancy, much less in the course of adult-adult relationships. 
Fortunately, missed opportunities can be remedied. Developments in secure base use after infancy are readily accessible to direct observation, as are corresponding changes in caregiver behavior. Figure 1 highlights key steps in the development of secure base behavior and representations from infancy through adolescence. The contexts and patterns of caregiver support that organize developing secure base use are on the left side of the table. These range from sensitive and cooperative interaction and explicit secure base teaching in infancy, to a supervision partnership beginning in early childhood, to service as an experienced listener and testing ground for emerging beliefs about self, others, and relationships beginning in middle childhood and early adolescence.

Key developments in secure base use and representation are on the right. These range from mere familiarity and preference, through an extended period of acquiring and consolidating secure base skills in infancy and toddlerhood. This is followed by a similarly extended period of formulating and consolidating representations of secure base experience, expectations, and skills through adolescence. The outline also incorporates the notion that experience with peers and early romantic partners play significant roles in this development.

Extending the time frame for attachment development and highlighting corresponding changes in caregiver behavior clarifies a number of points on which attachment theory is often misunderstood - particularly regarding openness to developmental change after infancy. It also highlights the strengths of the secure base concept as an organizational construct. Traditionally, attachment theory has been a theory of infancy and of adult relationships, with a great deal of what is in between left to the imagination. Completing this picture is essential to understanding the effects of early experience, the

\section{Figure 1 The Secure Base Phenomenon From Infancy to Adolescence}

\section{Relationship Contexts and Caregiver Support}

Sensitive, cooperative interaction

Caregiver monitors infant activities, retrieves, provides explicit secure base instruction

Practice, operant learning, improved locomotion, experience

Caregiver encourages independence, continued supervision

Caregiver explicitly summarizes secure base experience. Early co-construction of attachment representations.

Caregiver as experienced interlocutor; peers as ad hoc secure base figures; peer demands for secure base support

Caregivers' reactions to relationship decisions; parents' and peers' relationships as models

\section{Secure Base Behavior and Representations}

Familiarity, predictability, preference

Onset of secure base use

Secure base use consolidated

Secure base representation becomes portable, supervision partnership

Script-like representation of secure base experience

Applying representations of past experience to organize secure base use and service in romantic relationships; elaboration and consolidation of attachment representations

Discovery of implicit expectations, preferences, and sensitivities; reflection on successes and failures 
mechanisms underlying stability and change, and the relevance of ordinary socialization processes in attachment development (viz. Waters et al. 1991). For example, more emphasis should be placed on learning-related mechanisms, especially from the second year of life onward.

Extending the time frame for attachment development has important implications for longitudinal research. Specifically, it highlights the importance of including concurrent assessments of caregiver behavior in causal models of infant attachment and later development. Building severe tests of the mediating and moderating effects of caregiving and family dynamics into prospective, longitudinal designs is useful insurance against unstated premises and magical mechanisms slipping unnoticed into attachment theory.

\section{Attachment in Emergency and Ordinary}

\section{Circumstances}

Bowlby's emphasis on protection from predators as the evolutionary function of attachment has led current theorists and researchers to focus almost exclusively on attachment as an emergency response system. ${ }^{2}$ Moreover, the image of an infant fleeing to the safety of its mother's arms is one of the most evocative in human experience. However, this issue is also a continuing source of misunderstanding and missed opportunities for assessment, hypothesis testing, and new directions in attachment research.

For Bowlby and Ainsworth, the ability to use an attachment figure as a secure base affords a haven of safety and also provides the confidence necessary to explore and master ordinary environments. As currently formulated, attachment theory implies that a single control system appraises access to the caregiver and maintains a balance between proximity and exploration across both ordinary and emergency situations. This is supported by the fact that both emergency behavior in the Strange Situation and affect regulation in response to stressful attachment related stories are closely related to nonemergency attachment behavior at home
(Ainsworth et al, 1978; Vaughn \& Waters, 1990; Lay, Waters, Posada, \& Ridgeway, 1995). In addition, mothers' representations of attachment experiences in both ordinary and emergency situations strongly predict their ability to serve as a secure base for their children in ordinary free play situations (e.g., Posada, Waters, Crowell, \& Lay, 1995; Gao \& Waters, 1999).

Attachment in ordinary and emergency situations deserves high priority in both developmental and cross-cultural research. Evidence that they are consistently related would be strong support for Bowlby's notion of an underlying attachment control system. It would also open up a wide range of new possibilities for attachment assessment and for the effects of early experience. Evidence to the contrary would affect how we frame and test hypotheses about early experience. It might also require significant changes in current attachment theory.

\section{Expanding Horizons}

\section{The Child's Construction of Security}

As mentioned above, the attachment control system emerges from extended interplay of biases in human learning abilities with sensitive, cooperative care and secure base support. In infancy, experience with a particular caregiver can only be retained in the form of sensorimotor and sensori-affective representations. These forms of representation reflect only the behavioral possibilities and affective responses associated in experience with a particular person in a particular affective-behavioral context. They cannot be accessed voluntarily or in situations very different from the ones they reflect (viz. Sroufe, 1996). The symbolic representations of experience do not emerge until after infancy.

Bowlby was keenly aware that cognitive and conceptual development had implications for attachment after infancy. Even as he was detailing his ideas about attachment onset and development in infancy, he marked these issues for future study by including the goal corrected partnership in his developmental outline. 
Acquiring the capacity for symbolic representation has significant implications for (1) what defines a "secure base" and (2) the kinds of information that shape secure base expectations. The speech and behavior of toddlers and young children suggest that they (at least implicitly) construe "my parents", "my family", and perhaps even broader reference groups as sources of security. Better understanding these implications has become critical to developing the secure base concept for use beyond infancy and early childhood. The task is made easier and the prospects brighter by the successes of social cognition theory and research in developmental, social, and clinical psychology.

After infancy, observational learning too emerges as important influence on secure base expectations. The onset of social (observational) learning greatly expands the child's store of attachment-related information beyond what can be learned by direct interaction. On the positive side, noting skillful and consistent support of other family members can broaden a child's sense that its primary caregiver will be willing and able to serve as a secure base in any and all situations. On the negative side, unresolved family conflict and other evidence of adult's limitations can diminish a child's confidence in a caregiver's power or availability to provide support in difficult situations (viz., Cummings \& Davies, 1996).

Cognitive development also has important implications for understanding (1) the child's view of the family as a source of security, and (2) how, by early adolescence, friends, teachers, and other close relationships may be used selectively as secure base figures of convenience in specific contexts. Close attention to links between cognitive development and representations of secure base experience can play an important role in clarifying both the content and organization of attachment working models.

These are important issues. Unless we can define the content and organization of attachment working models rigorously, they soon take on properties as needed, push aside more parsimonious explanations, and ultimately explain too much (Hinde, 1988). Fortunately, as Bowlby had hoped, cognitive psychology today offers concepts and methods that can help us formulate and test a wide variety of hypotheses about the representation of early experience. Significant collaboration with cognitive and social psychologists is long overdue and would be a valuable theme for the next phase of attachment theory and research.

\section{Security in Family Context}

One of the cornerstones of modern developmental theory is the notion that dyadic relationships are nested within broader contexts (Bron fenbrenner, 1979). The family one of the most influential of these contexts. Bowlby (1949) was one of the first to call attention to the need to consider the family in understanding children's distress and security. Applying secure base and control system concepts to the family context can be a fertile source of new insights about relationships and development. For example, Cummings and Davies (1994) pointed out that infants and older children respond very differently to resolved versus unresolved family conflict. Even when both parents are readily accessible, they become distressed and often intervene in the unresolved conflicts - as if secure base access and coherent family relations are distinct sources of emotion regulation and security.

Consistent with this study and others on children's reactions to family stresses, Cummings and Davies (1996) proposed a control system model for children's security as a function of multiple family relationships and events. Within this model, a wide range of emotional, behavioral and representational processes are hypothesized to operate within an overarching goal of preserving emotional security. Davies and Cummings (1998) provided direct support for this model by demonstrating that children's emotional security about marital conflict mediates relations between marital conflict and child outcomes. This explicit theoretical model and emphasis on rigorous operationalization of constructs afford strong tests of the notion that security mediates links between family-wide functioning and child outcomes. 
Byng-Hall (1999) has also proposed an extension of the secure base notion to the family as a whole. Based on a clinical perspective, he has stressed the importance for children's well-being of a reliable family network and creating a secure family base, with a shared awareness among family members that attachments should protected and not undermined (see also Marvin \& Steward, 1990). Explorations of attachment in family context and the extension of the secure base construct to family-wide models is an important challenge for attachment theory. It is also an essential step toward realizing Bowlby's goal of significant clinical applications.

Secure Base Use and Secure Base Support in Adult Relationships

As mentioned above, the notion that across the life span close relationships are similar in kind was one of Freud's most daring speculations. Although rarely explicitly stated in current theory or research, it is reflected in the notion that attachment theory is relevant across the life span and in the notion that early relationship experience influences later development. The first step in testing this hypothesis is to apply Ainsworth's conceptualizations of secure base use and secure base support in infancy to adult's close relationships. Successfully measuring secure base use and support in adults would lend considerable support to attachment theory, provide a criterion against which to validate measures of adult attachment security, and help clarify the origins and functioning of close relationships in adulthood. Failure would undermine the notion that attachment theory is relevant across the life span, rendering it, perhaps, little more than a domain specific theory of infantcaregiver relationships.

Crowell, Gao, Pan, O'Connor, \& Waters (1997) have taken first steps in this direction by developing scales for assessing adults' skill at serving and using the partner as a secure base during 15 minute discussions of marital disagreements. Serving as a secure base is assessed in terms of criteria derived from Ainsworth et al's (1978) conceptualization of maternal sensitivity. It entails detecting the partner's implied or explicit requests for secure base support, correctly interpreting the request, and responding appropriately and in a timely manner. Secure base use is assessed in terms of criteria derived from Ainsworth et al.'s (1978) conceptualization of secure response in the Strange Situation. Key features include clearly signaling the need for secure base support, maintaining signals until they are detected, openness to the partner's response, and finding appropriate response comforting.

Preliminary results (Gao \& Waters, 1998) indicate that secure base use and support are indeed evident in couples' interactions. Moreover, as predicted from the notion that both are organized by the same secure base control system, they are significantly correlated. They are also significantly correlated with the coherence of attachment representations derived from the couples' experience with their own parents and their relationship to each other. ${ }^{3}$ These results provide preliminary support for the notion that close adult-adult relationships have significant secure base components.

Secure base use and support are not limited to the early phases of adult-relationships or to early adulthood. New demands for secure base use and opportunities to serve as a secure base present themselves throughout life. Figure 2 outlines a typical course of secure base use and support across the span of adulthood. As above, the contexts and patterns of support that organize secure base use in adulthood are on the left side of the figure and patterns of secure base use are on the right. This extended developmental outline opens up many new opportunities to study the influences of early experience on later attachment behavior. Particularly important is the opportunity to examine relations between early attachment experience and both secure base use and secure base support skills later in life and to examine them across contexts such as marriage, parenting, caring for adult parents, and requesting care from others. As currently formulated, attachment theory suggests that these are all organized by the same attachment control system and that skills would be corre- 


\section{Figure 2 The Secure Base Phenomenon in Adulthood}

\section{Secure Base Contexts and Partner Support}

Partner supports exploration and personal goals; partner requires secure base support

Transition to parenthood

Children require extended secure base support:

Emergencies; aging parents and partner

Aging; increased need for secure base support

\section{Secure Base Behavior and Representations}

Commitment in adult partnership; Secure base support of friends and junior partners (mentoring)

Close coordination with partner; balancing parenthood with other goals; drawing upon secure base experience to organize and motivate secure base support for partner and child

Serving as secure base for children; effectively requesting and using partner's (and parents') support in parenthood

Secure base service outside of parenting

Requesting and accepting secure base support from partner, children, and others lated across these domains. Empirical evidence on this point could either provide strong support for this formulation or suggest a more differentiated view of attachment representations.

The task of better assessing secure base use and support throughout adulthood deserves high priority in attachment research. Traditional views of adult close relationships focus primarily on processes in play when relationships are in distress. But just as peace is not merely the absence of war, the best close relationships offer adults more than well-regulated or benign conflicts. A secure base perspective on close relationships in adulthood offers a rich framework for conceptualizing and studying both troubled and successful relationships. The possibilities here are exciting and suggest new directions in research on adult development and marriage. It also suggests that attachment theory and research can serve as a useful organizational framework for prevention, intervention, and outcome research and eventually for clinical practice.

\section{Attachment Across Cultures}

Cross-cultural research on key issues in attachment theory is one of the most exciting prospects for the next generation of attachment research. However, at the present time the implications of attachment theory for attachment across cultures are widely misunderstood. Some of the misunderstanding can be traced to Bowlby's use of concepts and examples from classical ethology to support the notion that the kinds of learning biases necessary to account for an attachment control system can be shaped by natural selection. Bowlby's references to (1) attachment as part of out primate heritage, (2) imprinting and critical periods, (3) the importance of early care in the onset of attachment, (4) the relatively quick onset of attachment behavior in infancy, and (5) the enduring impact of early experience have led some to misconstrue and sometimes caricature his perspective as a modern instinct theory with strong assumptions about universality across cultures. These include 
the notion that the distribution of Strange Situation classifications should be similar across cultures.

In fact, close reading of Attachment and loss (Bowlby, 1969) supports a more sophisticated view. For Bowlby, the function of secure base relationships is always to support competence development and promote safety. This function is enhanced by access to (and confidence in) a sensitive and responsive secure base figure. Attachment theory does assume that sensitivity to infant signals, cooperative interaction, availability, and responsiveness play a role in attachment development. It does not assume that these are equally prevalent in every culture or community or that the distribution of Strange Situation classifications would be similar across cultures.

Moreover, the logic of modern attachment theory easily accommodates (1) the fact that the Strange Situation is not valid in every culture, (2) the use of multiple caregivers, (3) cultural influences, especially after infancy, on how caregiver availability and responsiveness are communicated, (4) communities or cultures in which attachment relationships are not the most salient factors in socialization and social adjustment, and (5) the fact that circumstances and social systems do not always give free rein to humans' capacity to form and maintain secure base relationships.

Thus, data on the distribution of Strange Situation classifications per se say little about the relevance of attachment theory acrosscultures. In contrast, studies examining the cross-cultural generality of (1) secure base behavior and (2) the relation of caregiver behavior to the organization of secure base behavior afford very useful tests of key attachment theory postulates. In the only study to have directly addressed the generality of secure base behavior, Posada, Gao, Wu, Posada, Tascan, Schoelmerich, Sagi, Kondo-Ikemura, Haaland, and Synnevag (1995) found evidence of secure base organization in all seven of the cultures they studied. In addition, Posada, Jacobs, Carbonell, Alzate, Bustamente, \& Arenas (1999) have recently reported strong correlations (.45-.60) be- tween maternal sensitivity and infant secure base behavior in samples of Colombian infants observed in home and hospital settings. These studies are models of cross-cultural research that closely track the logic of modern attachment theory and thus afford severe tests of key postulates. This is one of the most exciting directions in recent attachment theory and research and promises to bring cross-cultural perspectives back into the mainstream of attachment study.

\section{Attachment and Human Security}

As we enter this new era, attachment theorists seem more comfortable than ever exploring new directions. If we are mindful of the central role of the secure base concept and the control systems model, and put well-formed hypotheses to severe tests, the theory can continue to evolve without losing its coherence or over reaching. Pushing the secure base and control system concepts to their limits is part of fully understanding them and a source of new insights about relationships and development. It can also be a source of insights into the place of attachment in the grand scheme of things.

Although attachment theory captures important features of security provision across the life span, it doesn't exhaust them. Clearly there are many other assets and circumstances that can also contribute the sense of security (broadly construed). Although a responsive, available caregiver may be the primary source of security in infancy, by middle childhood, social relationships, athletic and academic competence, physical assets, and traits such as patience, bravery, or common sense can also be important sources of security. By adulthood, the list has expanded to include social alliances, status, and broad array of assets that are important across cultures (e.g., good health, experience, wealth) and culture specific assets (e.g., religious values, rank, and socially valued traits). Figure 3 outlines some of the sources of human security. The common thread among them it is that, in one way or another, each reduces uncertainty about the risks involved in exploration or in novel (including emergency) situations. The role of attachment figures in children's acquiring, concep- 
Figure 3 Some Sources of Human Security

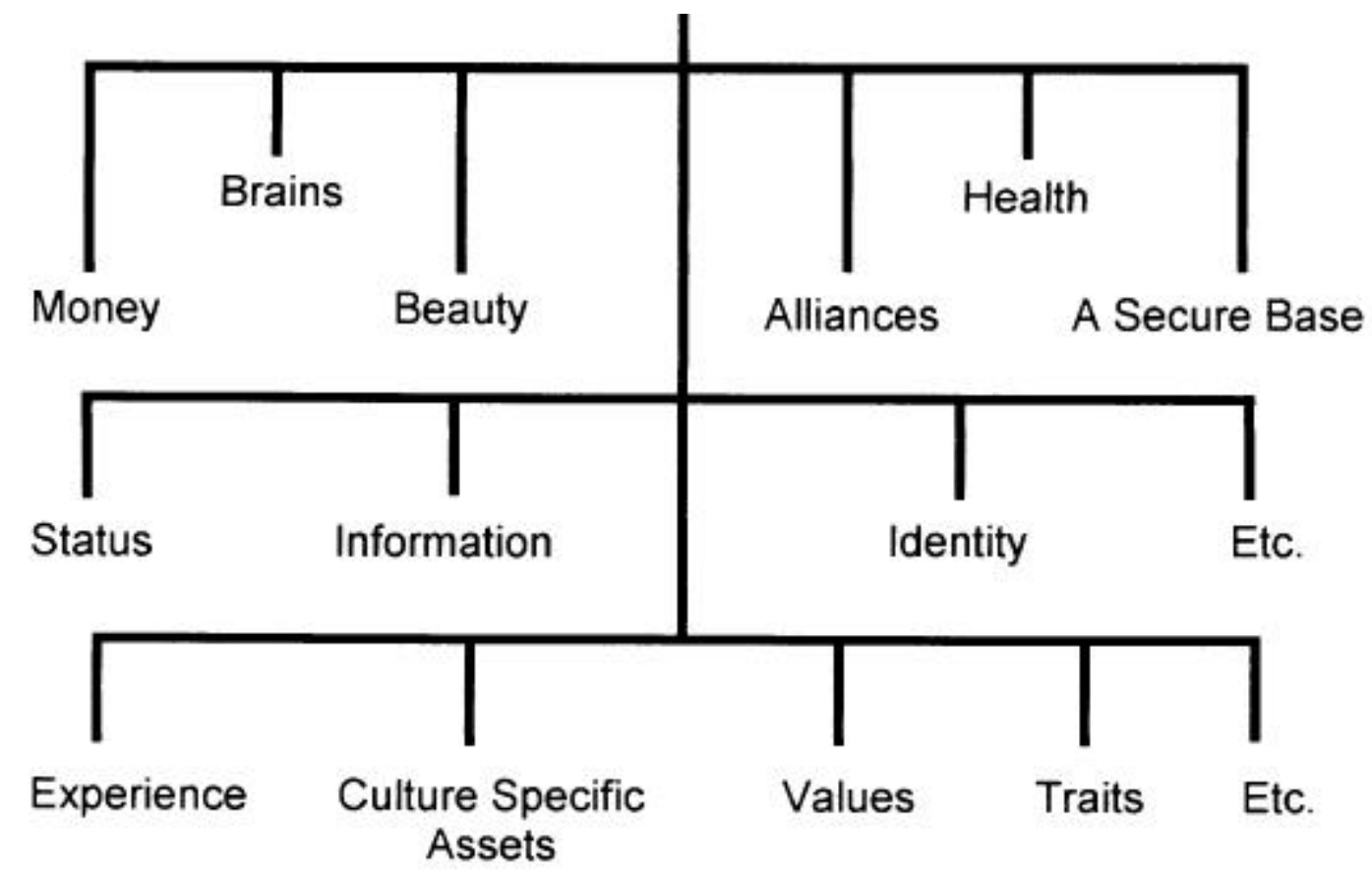

tualizing, and learning to effectively use such assets deserves high priority in research on socialization and personality development.

The fact that attachment relationships may be integral to many sources of human security does not imply that the Bowlby-Ainsworth perspective promises a general theory of human security. As we have mentioned, Bowlby's attachment concept refers to a very specific range of phenomena. It is about secure base use and secure base support in the context of close relationships that involve significant emotional bonds. It is too much to expect it to serve as a general framework for understanding human security or personality. Instead, the challenge is to find a scientific framework within which we can understand the many sources of human security, their interplay, and the roles of security in social development, personality, relationships, and adjustment. Although Bowlby's integrative approach to theory-building can serve as a model for first steps in this direction, the answer lies in placing attachment theory in the context of broader perspectives such as developmental psychopathology or cognitive self theory.

\section{Conclusion}

These are interesting times in attachment theory and research. For some, the issues we have raised are overdue; others may disagree. We have tried to keep to issues that can be addressed by explicating theoretical differences and putting them to empirical tests. This was a strength of Bowlby's and Ainsworth's approach to preserving Freud's insights about early experience and close relationships. It can serve equally 
well as we try to do for Bowlby and Ainsworth what they did for Freud - identify key insights, redress errors of analysis and emphasis, and enrich their legacy.

Psychology's grand theories rarely survive to celebrate a silver anniversary. In this era of specialization and domain specific theory, many have concluded that grand theory is unattainable or even discredited as a way of organizing and guiding empirical research (viz., McKinney, 1976). Nonetheless, its promise remains attractive. (e.g., Toews, 1985; Berscheid, 1995).

Measured from Bowlby's (1958) paper, "The nature of the child's tie to its mother", modern attachment theory is entering its fifth decade. With continued commitment to rigorous theoretical and empirical analysis, it promises to reach its silver anniversary more coherent and productive than ever, a fine legacy from John Bowlby and Mary Ainsworth that reflects well on developmental psychology and on the tradition of grand theory in the behavioral sciences.

\section{References}

Ainsworth, M.D.S. (1969). Object relations, dependency, and attachment: A theoretical review of the infant-mother relationship. Child Development, $\underline{40}, 969-1025$.

Ainsworth, M., Blehar, M., Waters, E., \& Wall, S. (1978). Patterns of attachment. Hillsdale, NJ.: Lawrence Erlbaum and Associates.

Berscheid, Ellen (1995). Help wanted: A grand theorist of interpersonal relationships, sociologist or anthropologist preferred. Journal of Social and Personal Relationships, 12, 529-533.

Bowlby (1949). The study and reduction of group tensions in the family. Human Relations, 2 , 123-128.

Bowlby, J. (1958). The nature of the child's tie to its mother. International Journal of Psychoanalysis, $\underline{39}, 350-373$.

Bowlby, I. (1969/1982). Attachment and loss (Vol. 1): Attachment. London, Hogarth Press.

Bretherton, I. \& Munholland, K. (1999). Internal Working Models in Attachment Relationships: A Construct Revisited. In J. Cassidy \& P.
Shaver (Eds. ), Handbook of attachment: Theory, research, \& clinical applications. (pp. 89-111). New York: Guilford Press.

Bronfenbrenner, U. (1979). The ecology of human development. Cambridge: Harvard University Press.

Byng-Hall, J. (1995). Creating a secure family base: Some implications of attachment theory for family therapy. Family Process, $\underline{34}$, 45-58.

Byng-Hall (1999). Family and couple therapy. In Cassidy, J. \& Shaver, P. (Eds. ), Handbook of attachment: Theory, research, and clinical applications (pp. 625-645). New York: Guilford Press.

Cassidy, J. \& Shaver, P. (Eds. ). (1999). Handbook of attachment: Theory, research, \& clinical applications. New York: Guilford Press.

Crowell, J., Gao, Y., Pan, H. \& Waters (1997). The secure base scoring system for adults (SBSS). Unpublished measure. Department of Psychology, SUNY, Stony Brook, NY 11794.

Crowell, J., Fraley, R. C., \& Shaver, P. (1999). Measurement of individual differences in adolescent and adult attachment. In J. Cassidy \& P. Shaver (Eds. ), Handbook of attachment: Theory, research, \& clinical applications. (pp. 434-465). New York: Guilford Press.

Crowell, J. \& Treboux, D. (1995). A review of adult attachment measures: Implications for theory and research. Social Development, 4, 294-327.

Cummings, E. M.; Davies, P. (1996). Emotional security as a regulatory process in normal development and the development of psychopathology. Development and Psychopathology, $\underline{8}$, 123-139.

Cummings, E. M. \& Davies, P. (1994). Children and marital conflict: The impact of family dispute and resolution. New York and London:

Davies, P. \& Cummings, E. M. (1998). Exploring children's emotional security as a mediator of the link between marital relations and child adjustment. Child Development, 69, 124-139.

Gao, \& \& Waters (1998, June). Secure base behavior and attachment security in engaged couples. Paper presented at the meeting of the International Society for the Study of Personal Relationships, Saratoga Springs, NY. 
Greenberg, M. Cicchetti, D., \& Cummings, M. (Eds.). (1990). Attachment in the preschool years: Theory, research, and intervention. Chicago: University of Chicago Press.

Hinde, R. (1988). Continuities and discontinuities. In M. Rutter (Ed.), Studies of psychosocial risk: The power of longitudinal data (pp. 367-384). Cambridge: Cambridge University Press.

Lay, K., Waters, E., \& Posada, G., \& Ridgeway, D. (1995). Attachment security, affect regulation, and defensive responses to mood induction. In E. Waters, B. Vaughn, G. Posada, and K. Kondo-Ikemura (Eds.) Caregiving, Cultural, and Cognitive Perspectives on Secure-Base Behavior and Working Models: New Growing Points of Attachment Theory and Research. Monographs of the Society for Research in Child Development, 60 (2-3, Serial no. 244), 179-196.

Marvin, R. \& Stewart (1990). A family systems framework for the study of attachment. In M. Greenberg, D. Cicchetti, \& M. Cummings (Eds.) Attachment in the preschool years: Theory, research, and intervention. (pp. 51-86). Chicago: University of Chicago Press.

Mayo, D. (1996). Error and the growth of experimental knowledge. Chicago: University of Chicago Press.

McKinney, Fred (1976). Fifty years of psychology. American Psychologist, 31, 834-842.

Meehl, P. E. (1978). Theoretical risks and tabular asterisks: Sir Karl, Sir Ronald, and the slow progress of soft psychology. Journal of Consult-

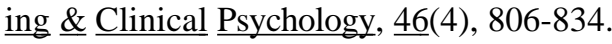

Posada, G., Jacobs, A., Carbonell, O. A., Alzate, G., Bustamante, M. R., \& Arenas, A. (1999) Maternal care and attachment security in ordinary and emergency contexts. Developmental Psychology. Vol. 35, 6, pp. 1379-1388.).

Posada, G., Gao, Y., Fang, W., Posada, R., Tascon, M., Schoelmerich, A., Sagi, A., KondoIkemura, K., Ylaland, W., \& Synnevaag, B. (1995) The Secure Base Phenomenon Across Cultures: Children's Behavior, Mothers' Preferences, and Experts' Concepts. In E. Waters, B. Vaughn, G. Posada, and K. Kondo-Ikemura (Eds.) Caregiving. Cultural and Cognitive Perspectives on Secure-Base Behavior and Work- ing Models: New Growing Points of Attachment Theory and Research. Monographs of the Society for Research in Child Development, 60 (2-3, Serial no. 244), 27-48.

Posada, G., Waters, E., Crowell, J., \& Lay, K. L. (1995). Is it easier to use a secure mother as a secure base: Attachment Q-sort correlates of the Berkeley Adult Attachment Interview. In E. Waters, B. Vaughn, G. Posada, and K. KondoIkemura (Eds.) Caregiving. Cultural and Cogni$\underline{\text { tive Perspectives }}$ on Secure-Base Behavior and Working Models: New Growing Points of Attachment Theory and Research. Monographs of the

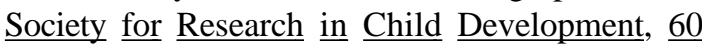
(2-3, Serial no. 244), 133-145.

Smedslund, J. (1994). What kind of propositions are set forth in developmental research? Five case studies. Human Development, 37, 280-292.

Sroufe, L. A. (1996). Emotional Development : The Organization of Emotional Life in the Early Years (Cambridge Studies in Social and Emotional Development). Cambridge: Cambridge University Press.

Sroufe, L. A. \& Waters E. (1977). Attachment as a organizational construct. Child Development, 48, 1184-1199.

Toews, John E. (1985). The return of grand theory in the human sciences. The American Historical Review, 92, 879-907

Vaughn, B. \& Waters, E. (1990). Attachment behavior at home and in the laboratory: Q-sort observations and Strange Situation classifications of oneyear-olds. Child Development, 61, 1965-1973.

Waters, E. (1997). The Attachment Q-set Advisor [On-line]. Available: http://www. psychology.sunysb.edu/ewaters/measures/aqs.htm.

Waters, E., Kondo-Ikemura, K., Posada, G. \& Richters, J. (1991). Learning to love: Mechanisms and milestones. In M. Gunner and Alan Sroufe (Eds.) Minnesota Symposia on Child Psychology: Vol. 23. Self Processes and Development. (pp. 217-255). Hillsdale, N.J.: Lawrence Erlbaum. [Available online: www.psychology.psy.sunysb.edu/ewaters]

Waters, E., Hamilton, C. \& Weinfield, N. (2000). The stability of attachment security from infancy to adolescence and early adulthood: General Introduction. Child Development (in press). 


\section{Footnotes}

1. Referred to hereafter as the prototype hypothesis.

2. In practice, we cannot know the evolutionary function of a behavior. As an historical fact, it is not open to empirical verification. The evolution of attachment reflects not the evolution of specific behaviors but of a control system that integrates them into a useful behavioral system. Although predator avoidance may have played a role in the evolution of specific behaviors, that is far too narrow a construction to place on the secure base phenomenon.

3. Social psychologists interested in adult attachment have also begin to relations between attachment security and secure base behavior in adults (viz. Crowell, Fraley, \& Shaver, 1999). 